\title{
Estimation of Target Behavior Tendencies using Dezert-Smarandache Theory *
}

\author{
Albena Tchamova \\ Central Laboratory for Parallel Processing \\ Bulgarian Academy of Sciences \\ "Acad. G. Bonchev" Str.,bl.25-A \\ 1113 Sofia, Bulgaria. \\ tchamova@bas.bg
}

\author{
Tzvetan Semerdjiev \\ Central Laboratory for Parallel Processing \\ Bulgarian Academy of Sciences \\ "Acad. G. Bonchev" Str.,bl.25-A \\ 1113 Sofia, Bulgaria. \\ signal@bas.bg
}

Jean Dezert
ONERA

29 Avenue de la Division Leclerc

92320 Châtillon, France.

Jean.Dezert@onera.fr

\begin{abstract}
This paper presents an approach for target behavior tendency estimation (Receding, Approaching). It is developed on the principles of DezertSmarandache theory (DSmT) of plausible and paradoxical reasoning applied to conventional sonar amplitude measurements, which serve as an evidence for corresponding decision-making procedures. In some real world situations it is difficult to finalize these procedures, because of discrepancies in measurements interpretation. In these cases the decision-making process leads to conflicts, which cannot be resolved using the well-known methods. The aim of the performed study is to present and to approve the ability of DSmT to finalize successfully the decision-making process and to assure awareness about the tendencies of target behavior in case of discrepancies in measurements interpretation. An example is provided to illustrate the benefit of the proposed approach application in comparison of fuzzy logic approach, and its ability to improve the overall tracking performance.
\end{abstract}

Keywords: DSmT, Data Fusion, Estimation, Uncertainty, Decision Making, Fuzzy Logic.

\section{Introduction}

Angle-only tracking systems based on sonars are poorly developed topic due to a number of complications. These systems tend to be less precise than

*This work is partially supported by MONT grants I-1205/02, I-1202/02 and by Center of Excellence BIS21 grant ICA1-200070016 those based on active sensors, but one important advantage is their vitality of being stealth. In a single sensor case only direction of the target as an axis is known, but the true target position and behavior (approaching or descending) remain unknown. Recently, the advances of computer technology lead to sophisticated data processing methods, which improve sonars capability. A number of developed tracking techniques operating on angle-only measurement data use additional information. In our case we utilize the measured emitter's amplitude values in consecutive time moments. This information can be used to assess tendencies in target's behavior and, consequently, to improve the overall angle-only tracking performance.

The aim of the performed study is to present and to approve the ability of DSmT to finalize successfully the decision-making process and to assure awareness about the tendencies of target behavior in case of discrepancies of angle-only measurements interpretation. Results are presented and compared with the respective results, but drawn from the fuzzy logic approach.

\section{Statement of the Problem}

In order to track targets using angle-only measurements it is necessary to compensate the unknown ranges by using additional information received from the emitter. In our case we suppose that in parallel with measured local angle the observed target emits constant signal, which is perceived by the sensor with 
a non-constant, but a varying strength (referred as amplitude). The augmented measurement vector at the end of each time interval $k=1,2, \ldots$ is $Z=\left\{Z_{\theta}, Z_{A}\right\}$, where: $Z_{\theta}=\theta+\nu_{\theta}$ denotes the measured local angle with zero-mean Gaussian noise $\nu_{\theta}=\mathcal{N}\left(0, \sigma_{\nu_{\theta}}\right)$ and covariance $\sigma_{\nu_{\theta}} ; Z_{A}=A+\nu_{A}$ denotes corresponding signal's amplitude value with zero-mean Gaussian noise $\nu_{A}=\mathcal{N}\left(0, \sigma_{\nu_{A}}\right)$ and covariance $\sigma_{\nu_{A}}$. The variance of amplitude value is because of the cluttered environment and the varying unknown distance to the object, which is conditioned by possible different modes of target behavior (approaching or descending). Our goal is, utilizing received amplitude feature measurement, to predict and to estimate the possible target behavior tendencies.

Figure 1 represents a block diagram of the target's behavior tracking system. Regarding to the formulated problem, we maintain two single-model-based Kalmanlike filters running in parallel using two models of possible target behavior - Approaching and Receding. At initial time moment $k$ the target is characterized by the fuzzified amplitude state estimates according to the models $A^{\mathrm{App}}(k \mid k)$ and $A^{\mathrm{Rec}}(k \mid k)$. The new observation $Z_{A}(k+1)=A(k+1)+\nu_{A}(k+1)$ is assumed to be the true value, corrupted by additive measurement noise. It is fuzzified according to the chosen fuzzification interface.

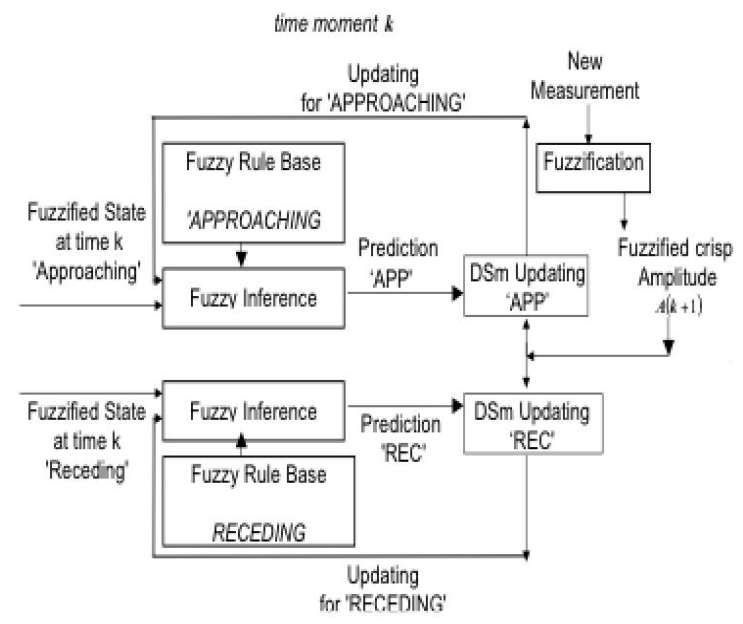

Figure 1: Block diagram of target's behavior tracking system

The tendency prediction approach is based on Zadeh compositional rule. The updating procedure uses Dezert-Smarandache combination rule to estimate target behavior states. Dezert-Smarandache Theory assures a particular framework where the frame of discernment is exhaustive but not necessarily exclusive and it deals successfully with rational, uncertain or paradoxical data. In general this diagram resembles the commonly used approaches in standard tracking systems $[1,2]$, but the peculiarity consists in the implemented particular approaches in the realizations of the main steps.

\section{Dezert-Smarandache Theory}

The practical limitations of the Dempster-Shafer Theory (DST) [10] come essentially from its inherent following constraints, which are closely related with the acceptance of the third exclude principle.

(C1) The DST considers a discrete and finite frame of discernment $\Theta$ based on a set of exhaustive and exclusive elementary elements $\theta_{i}$.

(C2) - the bodies of evidence are assumed independent and provide their own belief function on the powerset $2^{\Theta}$ but with same interpretation for $\Theta$.

In most of practical fusion applications based on the DST, some ad-hoc or heuristic techniques must always be added to the fusion process to manage or reduce the possibility of high degree of conflict between sources. Otherwise, the fusion results lead to a very dangerous conclusions or cannot provide a reliable results at all. To overcome these major limitations and drawbacks relative to the Dempster's rule of combination, a recent theory of plausible and paradoxical reasoning has been developed in $[3,11]$ and recently improved by Dezert in [4]. In general Dezert-Smarandache Theory (DSmT) can be considered as a generalization of the DST.

The foundations of the DSmT is to refute the principle of the third middle excluded and to allow the possibility for paradoxes (partial overlapping) between elements of the frame of discernment. The relaxation of the constraint $\mathrm{C} 1$ can be justified since the elements of $\Theta$ correspond generally only to imprecise/vague notions or concepts so that no refinement for satisfying $\mathrm{C} 1$ is actually possible (specially if natural language is used to described elements of $\Theta$ ). The DSmT refutes also the excessive requirement imposed by $\mathrm{C} 2$ since it seems clear that the frame is usually interpreted differently by the distinct sources of evidence (experts). Some subjectivity on the information provided by a source of information is almost unavoidable, otherwise this would assume, as within the DST, that all bodies of evidence have an objective/universal (possibly uncertain) interpretation or measure of the phenomena under consideration which unfortunately rarely (never) occurs in reality. Actually in most of cases, the sources of evidence provide their beliefs about some hypotheses only with respect to their own worlds of knowledge 
and experience without reference to the (inaccessible) absolute truth of the space of possibilities. The DSmT includes the possibility to deal with evidences arising from different sources of information which don't have access to absolute interpretation of the elements $\theta_{i}$ under consideration and can be interpreted as a general and direct extension of probability theory and the DST in the following sense. Let $\Theta=\left\{\theta_{1}, \theta_{2}\right\}$ be the simplest frame of discernment involving only two elementary hypotheses (with no more additional assumptions on $\theta_{1}$ and $\left.\theta_{2}\right)$, then

- the probability theory deals with basic probability assignments $m(.) \in[0,1]$ such that

$$
m\left(\theta_{1}\right)+m\left(\theta_{2}\right)=1
$$

- the DST deals with bba $m(.) \in[0,1]$ such that

$$
m\left(\theta_{1}\right)+m\left(\theta_{2}\right)+m\left(\theta_{1} \cup \theta_{2}\right)=1
$$

- the DSmT theory deals with new bba $m(.) \in[0,1]$ such that

$$
m\left(\theta_{1}\right)+m\left(\theta_{2}\right)+m\left(\theta_{1} \cup \theta_{2}\right)+m\left(\theta_{1} \cap \theta_{2}\right)=1
$$

\subsection{Hyper-Powerset and DSm rule}

Let $\Theta=\left\{\theta_{1}, \ldots, \theta_{n}\right\}$ be a set of $n$ elements which cannot be precisely defined and separated so that no refinement of $\Theta$ in a new larger set $\Theta_{\text {ref }}$ of disjoint elementary hypotheses is possible (we abandon here the Shafer's model). The hyper-powerset $D^{\Theta}$ is defined as the set of all composite propositions built from elements of $\Theta$ with $\cup$ and $\cap\left(\Theta\right.$ generates $D^{\Theta}$ under operators $\cup$ and $\cap$ ) operators such that

1. $\emptyset, \theta_{1}, \ldots, \theta_{n} \in D^{\Theta}$.

2. If $A, B \in D^{\Theta}$, then $A \cap B \in D^{\Theta}$ and $A \cup B \in D^{\Theta}$.

3. No other elements belong to $D^{\Theta}$, except those obtained by using rules 1 or 2 .

The cardinality of $D^{\Theta}$ is majored by $2^{2^{n}}$ when $\operatorname{Card}(\Theta)=|\Theta|=n$. The generation of hyper-power set $D^{\Theta}$ is closely related with the famous Dedekind's problem on enumerating the set of monotone Boolean functions. An algorithm for generating $D^{\Theta}$ based on isotone Boolean functions can be found in [5].

From a general frame of discernment $\Theta$, we define a map $m():. D^{\Theta} \rightarrow[0,1]$ associated to a given source of evidence $\mathcal{B}$ which can support paradoxical information, as follows

$$
m(\emptyset)=0 \quad \text { and } \quad \sum_{A \in D^{\Theta}} m(A)=1
$$

The quantity $m(A)$ is called $A$ 's generalized basic belief assignment (gbba) or the generalized basic belief mass for $A$. The belief and plausibility functions are defined in almost the same manner as within the DST, i.e.

$$
\begin{aligned}
& \operatorname{Bel}(A)=\sum_{B \in D^{\Theta}, B \subseteq A} m(B) \\
& \operatorname{Pl}(A)=\sum_{B \in D^{\Theta}, B \cap A \neq \emptyset} m(B)
\end{aligned}
$$

In the DSmT, it is not necessary to define the complementary proposition $\bar{A}$ of a proposition $A$ because of the refutation of the third middle excluded principle. These definitions are compatible with the DST ones when the sources of information become uncertain but rational. The DSm rule of combination $m(.) \triangleq$ $\left[m_{1} \oplus m_{2}\right]($.$) of two distinct (but potentially paradoxi-$ cal) sources of evidences $\mathcal{B}_{1}$ and $\mathcal{B}_{2}$ over $\Theta$ with belief functions $\operatorname{Bel}_{1}($.$) and \operatorname{Bel}_{2}($.$) associated with general$ information granules $m_{1}($.$) and m_{2}($.$) is given by [4],$

$$
\forall C \in D^{\Theta}, m(C)=\sum_{A, B \in D^{\Theta}, A \cap B=C} m_{1}(A) m_{2}(B)
$$

Since $D^{\Theta}$ is closed under $\cup$ and $\cap$ operators, this new rule of combination guarantees that $m():. D^{\Theta} \rightarrow[0,1]$ is a proper general information granule. This rule of combination is commutative and associative and can always be used for the fusion of paradoxical or rational sources of information (bodies of evidence). It is important to note that any fusion of sources of information generates either uncertainties, paradoxes or more generally both. The fusion process applied is justified from the maximum entropy principle.

\section{Approach for Behavior Ten- dency Estimation}

There are a few particular basic components in the block diagram of target's behavior tracking system.

\subsection{Fuzzification Interface}

A decisive variable in our task is the transmitted from the emitter amplitude value $A(k)$, received at consecutive time moments $k=1,2, \ldots$. We use the fuzzification interface (fig.2), that maps it into two fuzzy sets defining two linguistic values in the frame of discernments $\Theta=\{S \triangleq$ Small, $B \triangleq \operatorname{Big}\}$. Their membership functions are not arbitrarily chosen, but rely on the inverse proportion dependency between the measured amplitude value and corresponding distance to target.

The length of fuzzy sets' bases provide design parameter that we calibrate for satisfactory performance. These functions are tuned in conformity with the particular dependency $A \approx f(1 / \delta D)$ known as a priori 


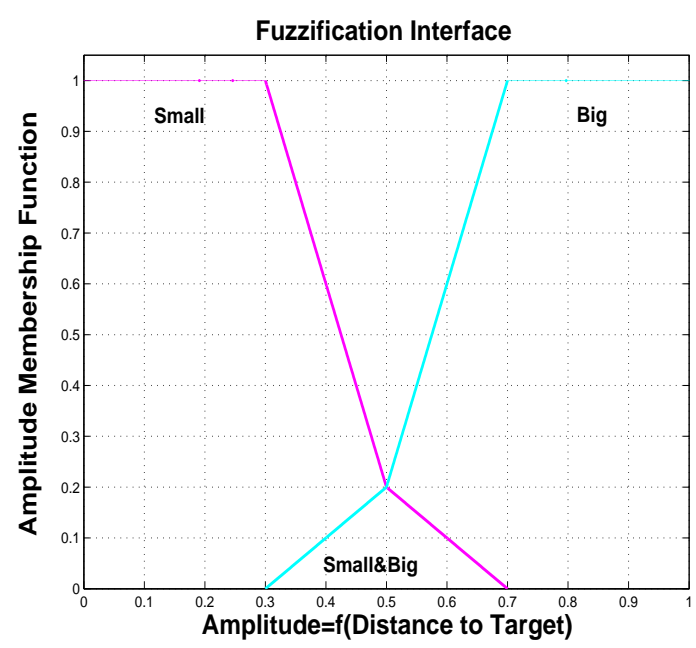

Figure 2: Fuzzification Interface

information The degree of overlap between adjacent fuzzy sets reflects amplitude gradients in the boundary points of specified distance intervals.

\subsection{Behavior Models}

In conformity with our task, fuzzy rules' definition is consistent with the tracking of amplitude changes tendency in consecutive time moments $k=1,2, \ldots$. With regard to this a particular feature is that considered fuzzy rules have one and the same antecedents and consequents. We define their meaning by using the prespecified in paragraph 4.1 linguistic terms and associated membership functions. We consider two essential models of possible target behavior:

Approaching Target - it's behavior is characterized as a stable process of gradually amplitude value increasing, i.e. the transition $S \rightarrow S \rightarrow$ $B \rightarrow B$ is held in a timely manner;

Receding Target - it's behavior is characterized as a stable process of gradually amplitude value decreasing, i.e. the transition $B \rightarrow B \rightarrow S \rightarrow S$ is held in a timely manner.

To comprise appropriately these models the following rule bases have to be carried out:

\section{Behavior Model 1: Approaching Target:}

Rule 1: IF $A(k)=S$ THEN $A(k+1)=S$

Rule 2: IF $A(k)=S$ THEN $A(k+1)=B$

Rule 3: IF $A(k)=B$ THEN $A(k+1)=B$

\section{Behavior Model 2: Receding Target:}

Rule 1: IF $A(k)=B$ THEN $A(k+1)=B$
Rule 2: IF $A(k)=B$ THEN $A(k+1)=S$

Rule 3: IF $A(k)=S$ THEN $A(k+1)=S$

The inference schemes for these particular fuzzy models are conditioned on the cornerstone principle of each modeling process. It is proven [9], that minimum and product inferences are the most widely used in engineering applications, because they preserve cause and effect. The models are derived as fuzzy graphs:

$$
g=\max _{i}\left(\mu_{A_{i} \times B_{i}}(u, v)\right)=\max _{i}\left(\mu_{A_{i}}(u) \cdot \mu_{B_{i}}(v)\right)
$$

in which $\mu_{A_{i} \times B_{i}}(u, v)=\mu_{A_{i}}(u) \cdot \mu_{B_{i}}(v)$ corresponds to the Larsen product operator for the fuzzy conjunction, $g=\max _{i}\left(\mu_{A_{i} \times B_{i}}\right)$ is the maximum for fuzzy union operator and $\mu_{B^{\prime}}(y)=\max _{x_{i}}\left(\min \left(\mu_{A^{\prime}}\left(x_{i}\right), \mu_{A \times B}\left(x_{i}, y_{i}\right)\right)\right)$ is the Zadeh max-min operator for the composition rule.

The fuzzy graphs related to the two models are obtained in conformity with the above described mathematical interpretations, by using the specified membership functions for linguistic terms Small, Big, and taking for completeness into account all possible terms in the hyper-Powerset $D^{\Theta}=\{S, B, S \cap B, S \cup B\}$ :

\begin{tabular}{|l|c|c|c|c|}
\hline$k \rightarrow k+1$ & $S$ & $S \cap B$ & $B$ & $S \cup B$ \\
\hline$S$ & 1 & 0 & 1 & 0 \\
\hline$S \cap B$ & 0 & 0 & 0 & 0 \\
\hline$B$ & 0.2 & 0 & 1 & 0 \\
\hline$S \cup B$ & 0 & 0 & 0 & 0 \\
\hline
\end{tabular}

Relation 1: Approaching Target

\begin{tabular}{|l|c|c|c|c|}
\hline$k \rightarrow k+1$ & $S$ & $S \cap B$ & $B$ & $S \cup B$ \\
\hline$S$ & 1 & 0 & 0.2 & 0 \\
\hline$S \cap B$ & 0 & 0 & 0 & 0 \\
\hline$B$ & 1 & 0 & 1 & 0 \\
\hline$S \cup B$ & 0 & 0 & 0 & 0 \\
\hline
\end{tabular}

Relation 2: Receding Target

\subsection{Amplitude State Prediction}

At initial time moment $k$ the target is characterized by the fuzzified amplitude state estimates according to the models $\mu_{A^{\mathrm{App}}}(k \mid k)$ and $\mu_{A^{\mathrm{Rec}}}(k \mid k)$. Using these fuzzy sets and applying the Zadeh max-min compositional rule [9] to relation 1 and relation 2, we obtain models' conditioned amplitude state predictions for time $k+1$, i.e. $\mu_{A^{\mathrm{App}}}(k+1 \mid k)$ is given by $\max \left(\min \left(\mu_{A^{\mathrm{App}}}(k \mid k), \mu_{\mathrm{App}}(k \rightarrow k+1)\right)\right)$ and $\mu_{A^{\mathrm{Rec}}}(k+$ $1 \mid k)$ by $\max \left(\min \left(\mu_{A^{\operatorname{Rec}}}(k \mid k), \mu_{\operatorname{Rec}}(k \rightarrow k+1)\right)\right)$. 


\subsection{State Updating using DSmT}

Dezert-Smarandache combinational rule is used here for state updating. This procedure is realized on the base of fusion between predicted states according to the considered models (Approaching, Receding) and the new measurement. Since $D^{\Theta}$ is closed under $\cup$ and $\cap$ operators, to obey the requirements to guarantee that $m():. D^{\Theta} \mapsto[0,1]$ is a proper general information granule, it is necessarily to transform fuzzy membership functions representing the predicted state and new measurement into mass functions. It is realized through their normalization with respect to the unity interval. Models' conditioned amplitude state prediction vector $\mu_{\mathrm{pred}}^{\mathrm{Ap} / \mathrm{Rec}}($.$) is obtained in the form:$

$$
\left[\mu_{\text {pred }}^{\mathrm{A} / \mathrm{R}}(S), \mu_{\text {pred }}^{\mathrm{A} / \mathrm{R}}(S \cap B), \mu_{\text {pred }}^{\mathrm{A} / \mathrm{R}}(B), \mu_{\text {pred }}^{\mathrm{A} / \mathrm{R}}(S \cup B)\right]
$$

In general the terms, contained in $\mu_{\text {pred }}^{\mathrm{App} / \mathrm{Rec}}$ represent the possibilities that the predicted amplitude behavior belongs to the elements of hyper powerset $D^{\Theta}$ and there is no requirement to sum up to unity. In order to use DSm combinational rule (1), it is necessary to make normalization over $\mu_{\mathrm{pred}}^{\mathrm{App} / \mathrm{Rec}}$ to obtain respective gbba $\forall C \in D^{\Theta}=\{S, S \cap B, B, S \cup B\}$ :

$$
m_{\text {pred }}^{\text {App } / \operatorname{Rec}}(C)=\frac{\mu_{\text {pred }}^{\text {App } / \operatorname{Rec}}(C)}{\sum_{A \in D^{\Theta}} \mu_{\text {pred }}^{\text {App } / \operatorname{Rec}}(A)}
$$

The equivalent normalization has to be made for the received new measurement before being fused with the DSm rule of combination.

\section{Example}

Let consider at scan 3 the predicted vector for the model Approaching $\mu_{\mathrm{pred}}^{\mathrm{App} / \mathrm{Rec}}(4 \mid 3)$ with components $\mu(S)=0.6, \mu(S \cap B)=0.15, \mu(B)=0.05$ and $\mu(S \cup B)=0.0$, then the normalization constant is $K=0.6+0.15+0.05+0.0=0.8$ and after normalization, one gets the resulting gbba

$$
\begin{gathered}
m_{\text {pred }}^{\text {App } / \operatorname{Rec}}(S)=\frac{0.6}{K}=0.75 \\
m_{\text {pred }}^{\text {App } / \operatorname{Rec}}(S \cap B)=\frac{0.15}{K}=0.1875 \\
m_{\text {pred }}^{\text {App } / \operatorname{Rec}}(B)=\frac{0.05}{K}=0.0625 \\
m_{\text {pred }}^{\text {App } / \operatorname{Rec}}(S \cup B)=\frac{0.0}{K}=0.0
\end{gathered}
$$

That way one can obtain $m_{\mathrm{pred}}^{\mathrm{App} / \mathrm{Rec}}($.$) as a general$ (normalized) information granule for the prediction of the target's behavior.
The target behavior estimate $m_{\mathrm{upd}}^{\mathrm{App} / \mathrm{Rec}}($.$) at mea-$ surement time is then obtained from $m_{\text {pred }}^{\mathrm{App} / \operatorname{Rec}}($.$) and$ the amplitude belief assignment $m_{\text {mes }}(B)$ (built from the normalization of the new fuzzyfied crisp amplitude measurement received) by the DSm rule of combination, i.e.

$$
\begin{aligned}
m_{\mathrm{upd}}^{\mathrm{App} / \operatorname{Rec}}(C) & =\left[m_{\mathrm{upd}}^{\mathrm{App} / \mathrm{Rec}} \oplus m_{\mathrm{mes}}\right](C) \\
& =\sum_{A, B \in D^{\Theta}, A \cap B=C} m_{\mathrm{pred}}^{\mathrm{App} / \operatorname{Rec}}(A) m_{\mathrm{mes}}(B)
\end{aligned}
$$

Since in contrast to the DST, DSmT uses a frame of discernment, which is exhaustive, but in general case not exclusive (as it is in our case for $\Theta=\{S, B\}$ ), we are able to take into account and to utilize the paradoxical information $S \cap B$. This information relates to the case, when the moving target resides in an overlapping intermediate region, when it is hard to predict properly the tendency in its behavior. Thus the conflict management, modeled that way contributes to a better understanding of the target motion and to assure awareness about the behavior tendencies in such cases.

\section{Decision criterion}

It is possible to build for each model $\mathrm{M}=$ (A)pproaching, (R)eceding a subjective probability measure $P_{\text {upd }}^{\mathrm{M}}($.$) from the bba m_{\text {upd }}^{\mathrm{M}}($.$) with the gen-$ eralized pignistic transformation (GPT) $[4,8]$ defined $\forall A \in D^{\Theta}$ by,

$$
P_{\mathrm{upd}}^{\mathrm{M}}\{A\}=\sum_{C \in D^{\Theta} \mid A \cap C \neq \emptyset} \frac{\mathcal{C}_{\mathcal{M}^{f}}(C \cap A)}{\mathcal{C}_{\mathcal{M}^{f}}(C)} m_{\mathrm{upd}}^{\mathrm{M}}(C)
$$

where $\mathcal{C}_{\mathcal{M}^{f}}(X)$ denotes the DSm cardinal of proposition $X$ for the free-DSm model $\mathcal{M}^{f}$ of the problem under consideration here [6]. The decision criterion for the estimation of correct model $M$ is then based on the evolution of the Pignistic entropies, associated with updated amplitude states:

$$
H_{p i g}^{\mathrm{M}}\left(P_{\text {upd }}^{\mathrm{M}}\right) \triangleq-\sum_{A \in \mathcal{V}} P_{\text {upd }}^{\mathrm{M}}\{A\} \ln \left(P_{\text {upd }}^{\mathrm{M}}\{A\}\right)
$$

where $\mathcal{V}$ denotes the parts of the Venn diagram of the model $\mathcal{M}^{f}$. The estimation $\hat{\mathrm{M}}(k)$ of correct model at time $k$ is given by the most informative model corresponding to the smallest value of the pignistic entropy between $H_{p i g}^{\mathrm{A}}\left(P_{\mathrm{upd}}^{\mathrm{A}}\right)$ and $H_{p i g}^{\mathrm{R}}\left(P_{\mathrm{upd}}^{\mathrm{R}}\right)$. 


\section{Simulation study}

A non-real time simulation scenario is developed for a single target trajectory (figure 3 ) in plane coordinates $X, Y$ and for constant velocity movement. The tracker is located at position $(0 \mathrm{~km}, 0 \mathrm{~km})$. The target's starting point and velocities are: $\left(x_{0}=5 \mathrm{~km}, y_{0}=\right.$ $10 \mathrm{~km}$ ), with following velocities during the two part of the trajectory $(\dot{x}=100 \mathrm{~m} / \mathrm{s}, \dot{y}=100 \mathrm{~m} / \mathrm{s})$ and $(\dot{x}=-100 m / s, \dot{y}=-100 m / s)$

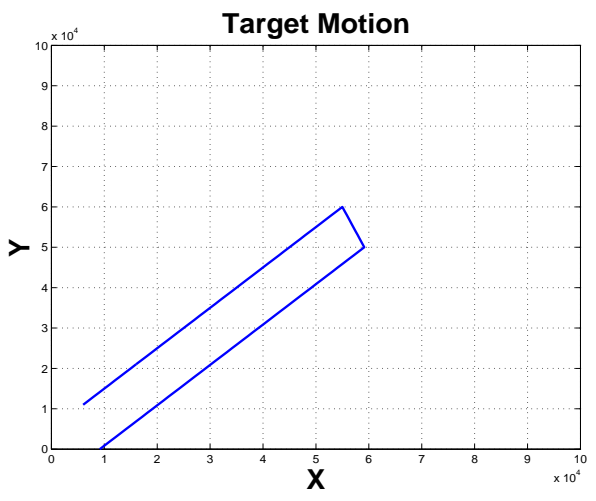

Figure 3: Target trajectory.

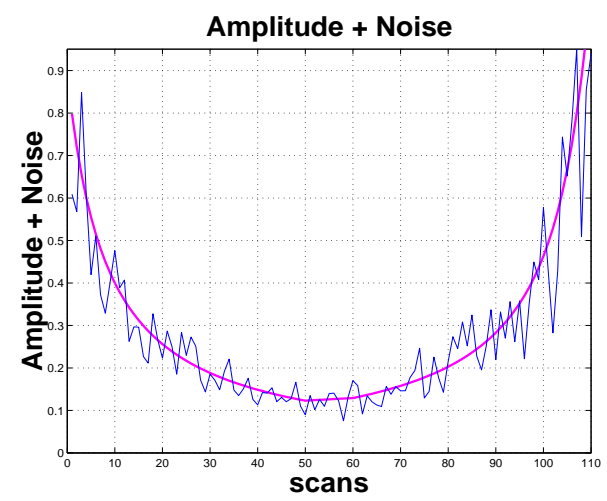

Figure 4: Measurements statistics.

The time sampling rate is $T=10 \mathrm{~s}$. The dynamics of target movement is modeled by equations:

$$
x(k)=x(k-1)+\dot{x} T \quad \text { and } \quad y(k)=y(k-1)+\dot{y} T
$$

The amplitude value $Z_{A}(k)=A(k)+\nu_{A}(k)$ measured by sonar is a random Gaussian distributed process with mean $A(k)=1 / D(k)$ and covariance $\sigma_{A}(k)$. $D(k)=\sqrt{x^{2}(k)+y^{2}(k)}$ is the distance to the target, $(x(k), y(k))$ is the corresponding vector of coordinates, and $\nu_{A}(k)$ is the measurement noise. Each amplitude value (true one and the corresponding noisy one) received at each scan is processed according to the block diagram (figure 1).

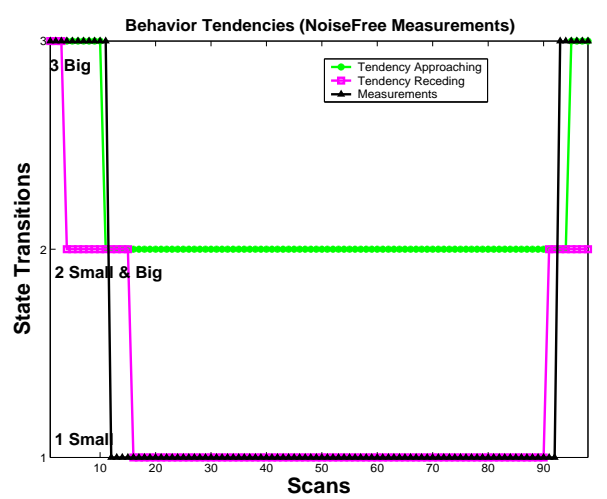

Figure 5: Behavior tendencies (Noise-free measurements).

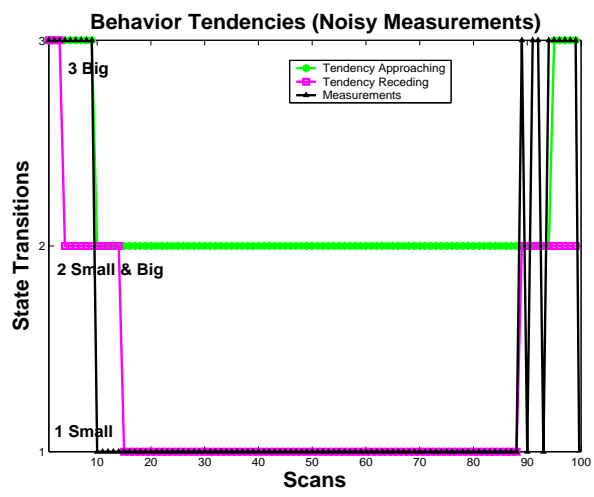

Figure 6: Behavior Tendencies (Noisy measurements).

Figures 5 and 6 show the results obtained during the whole motion of the observed target. Figure 5 represents the case when the measurements are without noise, i.e. $Z(k)=A(k)$. Figure 6 represents the case when measured amplitude values are corrupted by noise. In general the presented graphics show the estimated tendencies in target behavior, which are described via the scan consecutive transitions of the estimated amplitude states. Figure 7 represents the evolution of pignistic entropies associated with updated amplitude states for the Approaching and Receding models in case of noisy measurements (the figure for the noise-free measurement is similar and is not included here due to space limitation). It illustrates the decision criterion used to choose the correct model.

If one takes a look at the figure 5 and figure 7 , it can be seen that between scans 1st and 15th the target motion is supported by Approaching model, because that mode corresponds to the minimum entropies values, which means that it is the more informative one. The Approaching model is dominant, because the measured amplitude values during these scans stable reside in the state Big, as it is obvious from 


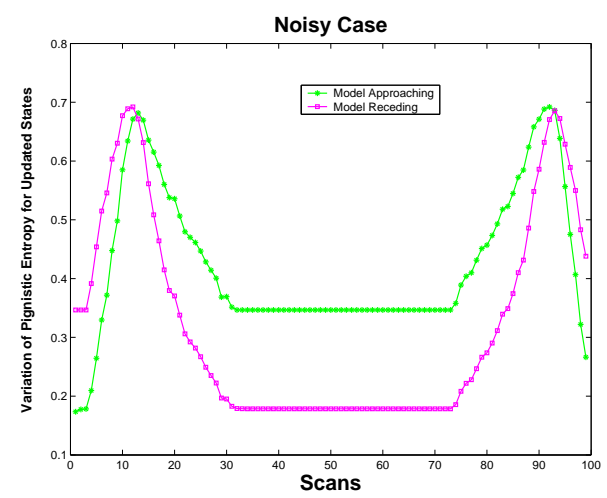

Figure 7: Evolution of the pignistic entropy for updated states.

the fuzzification interface (fig.2). In the same time, Receding model supports the overlapping region $S \cap B$, which is transition towards the state Small. Between scans 16th and 90th the Receding model becomes dominant since the variations of amplitude changes are minimal and their amplitude values stable support the state Small. During these scans Approaching model has a small reaction to the measurement statistics, keeping paradoxical state $S \cap B$.What it is interesting and important to note is that between scans 16 th and 30th the difference of entropies between Approaching and Receding models increases, a fact, that makes us to be increasingly sure that the Receding mode is becoming dominant. Then, between scans 75 th and 90th the difference of these entropies is decreasing, which means that we are less and less sure, that Receding model remain still dominant. After switching scan 91th the Approaching model becomes dominant one, until scan 100th. In general the reaction of the considered models to the changes of target motion is not immediate, because the whole behavior estimation procedure deals with vague propositions Small, Big, and sequences of amplitude values at consecutive scans often reside stable in one and the same states.

Comparing the results in fig. 6 with the results in figure 5 , it is evident, that although some disorder in the estimated behavior tendencies, one can make approximately correct decision due to the possibility of DSmT to deal with conflicts and that way to contribute for a better understanding of target behavior and evaluation of the threat.

\section{Comparison between DSm and Fuzzy Logic Approaches}

The objective of this paragraph is to compare the results received by using DSm theory and respective results but drawn from the Fuzzy Logic Approach (FLA) [9, 12, 13], applied on the same simulation scenario. The main differences between the two approaches consist in the domain of considered working propositions and in the updating procedure as well. In present work, we use DSm combination rule to fuse the predicted state and the new measurement to obtain the estimated behavior states, while in the fuzzy approach state estimates are obtained through a fuzzy set intersection between these entities. It is evident from the results, shown in figures 8 and 9 , that here we deal with only two propositions $\Theta=\{$ Small, Big $\}$. There is no way to examine the behavior tendencies in the overlapping region, keeping into considerations every one of possible target's movements: from $S \cap B$ to $B$ or from $S \cap B$ to $S$.

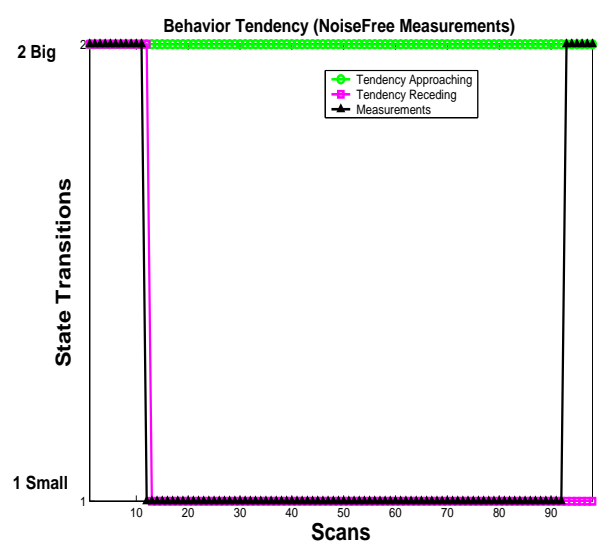

Figure 8: Behavior Tendencies (NoisyFree Measurements) drawn from Fuzzy Logic Approach.

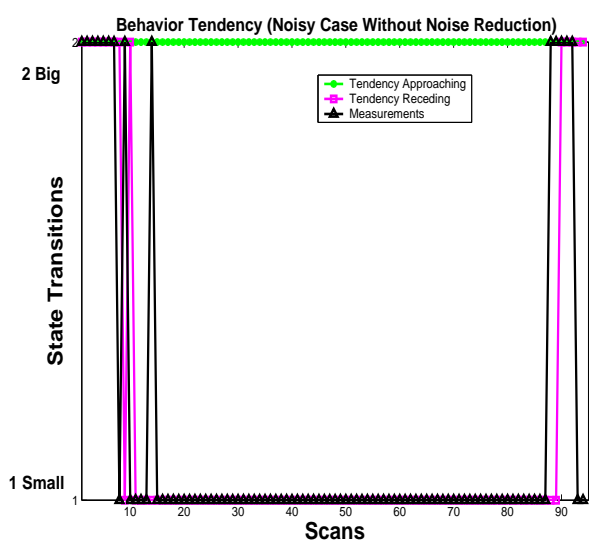

Figure 9: Behavior Tendencies (Noisy Case) without Noise Reduction drawn from Fuzzy Logic Approach.

Figure 8 shows the noise-free measurement case. It could be seen that between scan 10 and 90 target mo- 
tion is supported by the correct for that case Receding model, while Approaching one has no reaction at all. If we compare corresponding figure 5 (DSm case) and present figure 8, we can see, that in the case of DSm approach Receding model reacts more adequately to the true target tendency, because there is a possibility to deal with the real situation - the tendency of the target to make a movement from $B$ to the overlapping region $B \cap S$. In the FLA case there is no such opportunity and because of that between scan 1st and 10th Receding model has no reaction to the real target movement towards the $B \cap S$. Figure 9 represents the case when the measured amplitude values are corrupted by noise. It is difficult to make proper decision about the behavior tendency, especially after scan 90th., because it is obvious, that here the model Approaching coincide with the model Receding. In order to reduce the influence of measurement noise over tendency estimation, an additional noise reduction procedure has to be applied to make the measurements more informative. Its application improves the overall process of behavior estimation. Taking in mind all the results drawn from DSmT and FLA application, we can make the following considerations:

- DSmT and FLA deal with a frame of discernment, based in general on imprecise/vague notions and concepts $\Theta=\{S, B\}$. But DSmT allows us to deal also with rational, uncertain or paradoxical data, operating on the hyper powerset $D^{\Theta}=\{S, S \cap$ $B, B, S \cup B\}$. In our particular application it gives us an opportunity for flexible tracking the changes of possible target behavior during the overlapping region $S \cap B$.

- DSmT based behavior estimates can be characterized as a noise resistant, while FLA uses an additional noise reduction procedure to produce 'smoothed' behavior estimates.

\section{Conclusions}

An approach for estimating the tendency of target behavior was proposed. It is based on DezertSmarandache theory applied to conventional sonar measurements. It was evaluated using computer simulation. The provided example illustrates the benefits of DSm approach in comparison of fuzzy logic one. Dealing simultaneously with rational, uncertain and paradoxical data, an opportunity for flexible and robust reasoning is realized, overcoming the described limitations relative to the fuzzy logic approach. It is presented and approved the ability of DSmT to ensure reasonable and successful decision-making procedure about the tendencies of target behavior in case of discrepancies of angle-only measurements interpretation. The proposed approach yields confident picture for complex and ill-defined engineering problems.

\section{References}

[1] Bar-Shalom Y. (Ed.), Multitarget-Multisensor Tracking: Advanced Applications, Norwood, MA, Artech House, 1990.

[2] Blackman S., Popoli R., Design and Analysis of Modern Tracking, Artech House, 1999.

[3] Dezert J., Foundations for a new theory of plausible and paradoxical reasoning, Inform. \& Security, An int. Journal, Tzv. Semerdjiev Editor, CLPP, Bulg. Acad. of Sciences, Vol. 9, 2002.

[4] Dezert J., Fondations pour une nouvelle théorie du raisonnement plausible et paradoxal, ONERA Tech. Rep. 1/06769 DTIM, Jan. 2003.

[5] Dezert J., Smarandache F., On the generation of hyper-powersets for the DSmT, Proc. of Fusion 2003 Conf., Cairns, Australia, July 8-11, 2003.

[6] Dezert J., Smarandache F., Partial ordering of hyper-powersets and matrix representation of belief functions within DSmT, Proc. of Fusion 2003 Conf., Cairns, Australia, July 8-11, 2003.

[7] Dezert J., Smarandache F., Tchamova A., On the Blackman's association problem, Proc. of Fusion 2003 Conf., Cairns, Australia, July 8-11, 2003.

[8] Dezert J., Smarandache F., Daniel M., On the generalized pignistic transformation based on $D S m T$ framework, (in preparation), 2003.

[9] Mendel J.M., Fuzzy Logic Systems for Egineering: A Tutorial, Proc. of the IEEE, pp. 345-377, March 1995.

[10] Shafer G., A Mathematical Theory of Evidence, Princeton Univ. Press, Princeton, NJ, 1976.

[11] Smarandache F. (Editor), Proc. of the 1st Int. Conf. on Neutrosophics, Univ. of New Mexico, Gallup Campus, NM, USA, 1-3 Dec. 2001, Xiquan, Phoenix, 2002.

[12] Zadeh L., Fuzzy Sets as a Basis for a Theory of Possibility, Fuzzy Sets and Systems 1, pp.3-28, 1978 .

[13] Zadeh L., From Computing with Numbers to Computing with Words - From Manipulation of Measurements to Manipulation of Perceptions, IEEE Trans. on Circuits and Systems 45,1, pp.105-119, Jan. 1999. 